Open Access

Original Article

\title{
Influence of contemporary CAD-CAM milling systems on the fit and adaptation of partially stabilized Zirconia fixed partial dentures
}

\author{
Khulud A Al-Aali ${ }^{1}$, Rana S Alhamdan ${ }^{2}$, \\ Ahmed M Maawadh ${ }^{3}$, Fahim Vohra ${ }^{4}$, Tariq Abduljabbar ${ }^{5}$
}

\begin{abstract}
Objective: To evaluate marginal fit and internal adaptation of three-unit $\mathrm{Zr}$ frameworks fabricated from four $\mathrm{Zr}$ CAD/CAM milling systems.

Methods: Fixed partial denture models were replicated (40 stone models) using Polyvinyl Siloxane impression material (PVS) and type IV stone for Zr framework fabrication. FPDs were milled with four CAD/CAM systems, Group-II: LAVA ${ }^{\mathrm{TM}}$ Zirconia milled by LAVA ${ }^{\mathrm{TM}}$, Group-2: Vita In-Ceram YZ milled by Cerec ${ }^{\circledR}$, Group-3: Zirconia milled by GM1000 and Group-4: Zirconia milled by DWX-50N. Twelve marginal gap measurements per framework were performed at pre-established points, with a metallurgical microscope (Zeiss, Germany) at 500X magnification. Eight measurements of cement space per section were performed for adaptation. Data was analyzed using ANOVA and Tukey post hoc test.

Results: Zirconia FPD frameworks exhibited gaps ranging from 16 to $50.1 \mu \mathrm{m}$ for marginal fit and 26.8 to $102.5 \mu \mathrm{m}$ for internal adaptation. Group-3 [20.8 (8.3) $\mu \mathrm{m} \& 50.3(11.4) \mu \mathrm{m}]$ and Group-4 [16.0 (4.0) $\mu \mathrm{m} \&$ $40.2(8.8) \mu \mathrm{m}]$ specimens showed significantly lower marginal fit and internal adaptation gaps compared to Group-I [50.1 (13.4) $\mu \mathrm{m}$ \& $100.5(16.7) \mu \mathrm{m}$ ] and Group-2 [38.9 (8.2) $\mu \mathrm{m}$ \& 102.5 (13.4) $\mu \mathrm{m}$ ] specimens respectively.

Conclusions: Different CAD-CAM systems for fabrication of Zr FPD frameworks displayed a significant influence on marginal fit and internal adaptation of restorations.
\end{abstract}

KEYWORDS: Marginal fit; Internal adaptation; CAD-CAM; Milling parameters; Fixed partial dentures.

How to cite this:

doi: https://doi.org/10.12669/pjms.37.1.3490

Al-Aali KA, Alhamdan RS, Maawadh AM, Vohra F, Abduljabbar T. Influence of contemporary CAD-CAM milling systems on the fit and adaptation of partially stabilized Zirconia fixed partial dentures. Pak J Med Sci. 2021;37(1):45-51. doi: https://doi.org/10.12669/pjms.37.1.3490

This is an Open Access article distributed under the terms of the Creative Commons Attribution License (http://creativecommons.org/licenses/by/3.0), which permits unrestricted use, distribution, and reproduction in any medium, provided the original work is properly cited.

Correspondence:

Fahim Vohra

Prosthetic Dental Science Department,

College of Dentistry,

King Saud University,

Riyadh 11545,

Saudi Arabia.

Research Chair for Biological Research in Dental Health,

King Saud University, Riyadh,

Saudi Arabia.

Email: fvohra@ksu.edu.sa

* Received for Publication:

* Revision Received:

* Revision Accepted:
August 17, 2020

October 15, 2020

October 25, 2020

\section{INTRODUCTION}

There has been a paradigm shift in the field of dentistry, especially with the growing awareness for high-quality cosmetic dentistry and biocompatibility. ${ }^{1}$ This inspired development of new dental ceramics for single crowns, fixed partial denture, and implant restorations to provide alternative treatment options. In addition to the advanced improvement in the mechanical properties of the dental ceramic materials, new sophisticated processing technologies and systems have been introduced for the production of all-ceramic restorations. ${ }^{2}$ Different constructing 
techniques used to fabricate ceramic restorations include, hot-pressing, slip-casting, and computeraided design-computer aided manufacturing (CAD-CAM) systems.

CAD-CAM systems employ contemporary technologies for milling restorations from densely sintered or partially sintered ceramic blocks. ${ }^{3}$ A scanner digitizes the prepared tooth or die and the restoration is fabricated according to the previously established design. However, contemporary CADCAM systems do present shortcomings like the use of reflective powder coating before scanning of the tooth margins causing restorative distortion. ${ }^{4}$ In addition, CAD/CAM machining may lead to chipping defects, surface flaws and micro cracking due to the grinding processes. Such defects not only affect the fit of the restoration, but may also reduce its mechanical strength and clinical prognosis. ${ }^{5}$

One critical criterion for the success of CAD-CAM manufacturing systems is the marginal integrity and internal fit accuracy of the restorations. Accurate fit of restorations is extremely essential to achieve acceptable longevity. ${ }^{6}$ The presence of discrepancies at the crown margins favors plaque accumulation and microleakage, dissolution of cement, leading to secondary caries and periodontal disease. ${ }^{6}$ Multiple studies have assessed the marginal fit and adaptation of CAD-CAM restorations. ${ }^{7}$ It is suggested that the marginal accuracy of restorations made with CADCAM systems may be influenced by the designing software's and parameters. ${ }^{8}$

It is reported that parameters specific to the milling and machining process including number and dimensions of burs, axis of milling and machining type (soft or hard) may effect the fit and adaptation of CAD-CAM restorations. ${ }^{9,10}$ A three or four axis machine may not be capable of producing undercuts, therefore show low accuracy and marginal fit. ${ }^{11}$ Furthermore, milling of partially stabilized zirconia in dry conditions has shown better restorative accuracy then wet milling. ${ }^{10}$ However, in a study by Kirsch et al, restoration fabricated with a 4-axis CEREC milling machine revealed comparable fit outcomes to restoration made with 5 -axis milling machines. ${ }^{12}$ Therefore a controversy exists in these findings. It is hypothesized that partially stabilized zirconia restorations when fabricated using different CADCAM milling machines and systems will show a significant difference in their marginal fit and adaptation. Therefore, the aim of the study was to compare the marginal fit and internal adaptation of partially stabilized zirconia fixed partial dentures fabricated with different CAD-CAM systems.

\section{METHODS}

This experimental study was performed over a period of twelve months and reported inline with the checklist for reporting in-vitro studies (CRIS). The project was approved by the ethics and research committee at specialist practice and research center. (Ref. FR-031 June 21 ${ }^{\text {st }}$ 2019)

Preparation of master model: Acrylic resin teeth (second premolar and second molar) were mounted in clear EpoxiCure ${ }^{\circledR}$ Epoxy Resin (Buehler, USA) with space for a missing first molar tooth to form the master model (Fig.1-A). The mounted teeth were prepared for all-ceramic zirconia fixed partial denture (FPDs) restorations followed preparation guidelines by Charles Goodacre et al. with features of deep chamfer finish lines, $2 \mathrm{~mm}$ functional

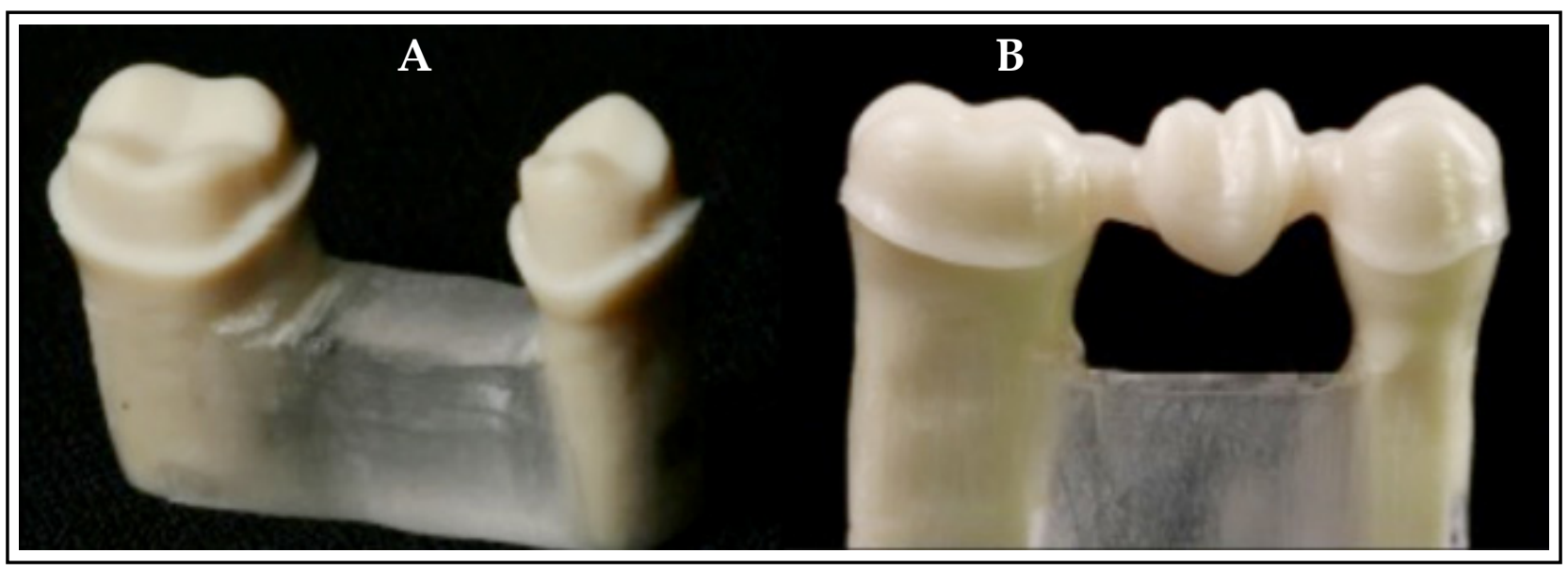

Fig.1. Prepared teeth model for scanning (A); Partially stabilized Zr milled framework (B). 
cusp bevel, $1.5 \mathrm{~mm}$ axial surface and $10^{\circ}-20^{\circ}$ total occlusal convergence angle. A carbide-finishing bur was used to finish the entire preparation. The model teeth were cleaned using a steamer.

Impression and preparation of master dies: Using polyvinyl siloxane impression material (Reprosil, DentsplySirona, MI, USA) in sectioned stock tray, the "master model" was impressed with a light body material at the finish line and heavy body material in the sectioned tray. After 5-6 minutes of setting, each impression was examined for defects or air bubbles. A total of 40 impressions were made.

All impressions were carefully boxed with boxing wax (Kerr). Thirty impressions were poured with type IV stone (Whip mix), generating thirty stone models for fixed partial denture (FPDs) frameworks fabrication. The other ten impressions were poured with scan able stone (Diamond die, HI-Tec) according to manufacture's instructions (liquid/powder ratio, 19 to $20 \mathrm{cc}$ water to 100 grams of powder). Powder was added gradually to water and vacuum mixed for 20 to 40 seconds. A total of 40 models were fabricated.

Fabrication of zirconia frameworks: Partially stabilized Zirconia fixed partial denture (FPD's) frameworks were milled for all models (forty) using four CAD-CAM systems. This resulted in four study groups based on the type of manufacturing techniques and systems $(n=10)$. A total of forty zirconia frameworks were fabricated using the recommended materials and following the manufacturers instructions (Fig.1-B). The four systems used are as follows:

Group-II: LAVA ${ }^{\mathrm{TM}}$ Zirconia $\left(3 \mathrm{M}^{\mathrm{TM}}\right.$ ESPE, US) frameworks milled by LAVA ${ }^{\mathrm{TM}}$ CNC 240.

Group-2: Vita In-Ceram YZ (VITA, Germany) frameworks, scanned with InEos Red Scan (Sirona Dental Systems, Germany), milled with Cerec ${ }^{\circledR}$ inLab (Sirona Dental Systems, Germany).

Group-3: Aadva ${ }^{\mathrm{TM}}$ Zirconia (Zr) (GC Advanced technologies Inc.) frameworks milled by GM1000 (GC Advanced technologies Inc.).

Group-4: Katana Zirconia (Noritake®, Japan) frameworks milled by DWX-50N (Noritake ${ }^{\circledR}$, Japan).

Marginal fit measurements: Each framework was secured on the respective stone model with a special clamp with uniform force at the pontic area. A metallurgical microscope (Zeiss, Germany) connected to a high precision digital videomicrometer (Javelin JV6000, California, USA) was used as for marginal gap measurements at 500x magnification. A total of twelve marginal gap measurements per framework were performed at pre-established points, six (6) measurements per tooth "mesio-buccal, mid-buccal, disto-buccal, mesio-lingual, mid-lingual, disto-lingual" for both prepared abutment teeth (Fig.2-A). The images of the specimens measured were saved as bit-map (bmp) files on the Windows ${ }^{\circledR}$ based computer attached to the microscope. The specimens were positioned in away that the margins were vertical on the measuring panel allowing the vertical lines in the video-micrometer to measure the gap (Fig.2-A). The marginal fit and internal adaptation testing was performed by single operator (KAA)

Internal fit for zirconia CAD/CAM frameworks: All frameworks were cemented on respective stone die using dual cure resin cement RelyX ${ }^{\mathrm{TM}}$ Unicem Aplicap $^{\mathrm{TM}}$ (3M ESPE). Cementation was done individually and one Rely $X^{\mathrm{TM}}$ Unicem Aplicap ${ }^{\mathrm{TM}}$ capsule was used per framework. The RelyX ${ }^{\mathrm{TM}}$ Unicem Aplicap ${ }^{\mathrm{TM}}$ was activated and triturated

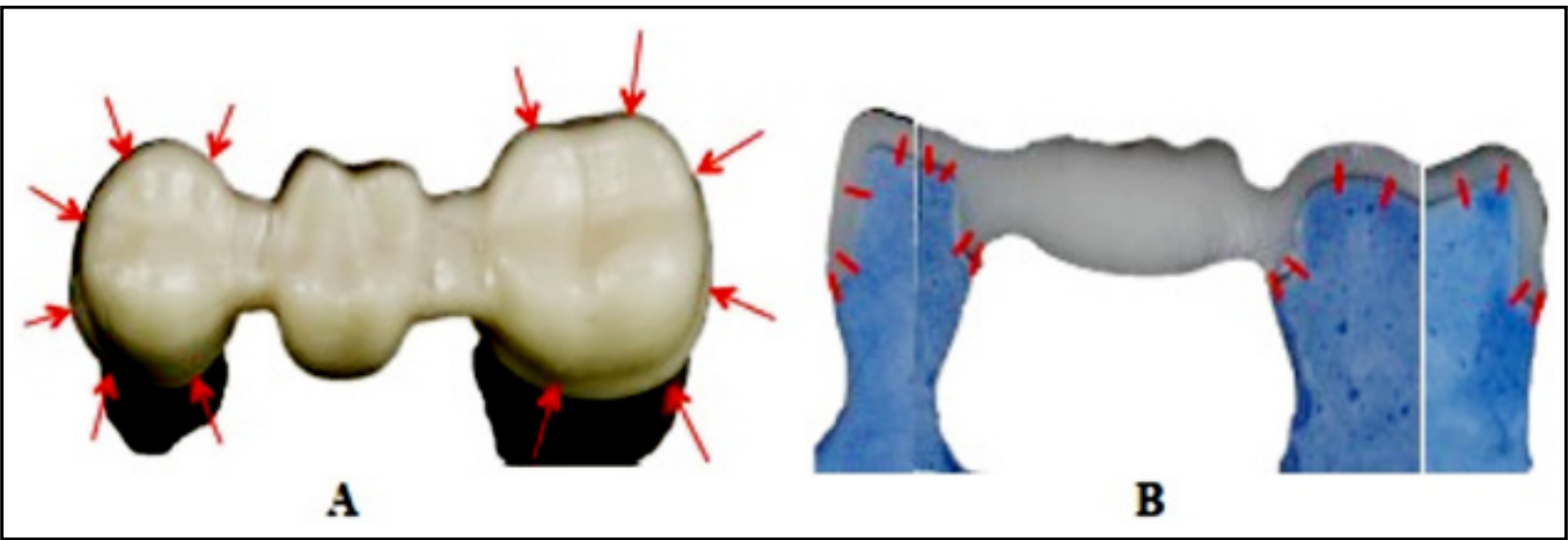

Fig.2: Point locations for measurements of marginal gap (A) and internal adaptation

(B) of fixed partial denture frameworks. 
for 15 seconds $(4,300$ RPM) utilizing a 3M ESPE CapMix ${ }^{\mathrm{TM}}$ Triturator according to manufacturer's instructions. The cement was dispensed into the framework and then placed on the die. A weight device axially loaded frameworks on the dies at $50 \mathrm{~N}$ for 10 minutes with the metal ball centrally at the occlusal part of the pontic. Excess cement was cleaned and allowed to auto cure (eight minutes). All cemented frameworks were carefully boxed (boxing wax-Kerr) and embedded using clear epoxy resin (EpoxiCure ${ }^{\circledR}$ Epoxy Resin- Buehler, USA). Specimens were sectioned (Isomet ${ }^{\circledR} 2000$ Precision diamond saw- Buehler, USA) under 200 grams load at a speed of $500 \mathrm{rpm}$ under copious irrigation, mesio-distally and bucco-lingually through the center of abutment teeth, dividing each specimen six parts. (Fig.2-B). The finished and polished (Buehler grinding-polishing system-Buehler Ltd) specimens were assessed at eight pre-established measurements of cement space for both abutment teeth (bucco-lingual and mesio-distal). The specimens were secured to metal rectangular plates attached with grey modeling clay and placed in the metallurgical microscope (Zeiss, Germany) connected to a high precision digital video-micrometer (Javelin JV6000, California, USA). Measurements were made at 500x magnification with the flat surface of the specimen facing the optical lens. Eight pre-established measurements (Fig.2-B) of cement space per section were performed for both abutment teeth for both specimen sides mesio-distal and bucco-lingual giving 16 gap measurements for each framework. Resulting in a total of 160 readings being measured for each system (group). Both marginal fit and internal adaptation were expressed in microns $(\mu \mathrm{m})$. Means and standard deviations among the study groups were compared employing ANOVA and Tukey Kramer multiple comparisons test. Some parts of the methodology were adopted from previous studies. $^{3}$

\section{RESULTS}

The maximum and minimum marginal fit gap was observed in Group-II $(50.1 \pm 13.4 \mu \mathrm{m})$ (LAVA ${ }^{\text {TM }}$ Zirconia- LAVA ${ }^{\mathrm{TM}}$ CNC 240$)$ and group-4 (16.0 \pm $4.0 \mu \mathrm{m})$ (Katana Zirconia-DWX-50N) specimens respectively (Table-I). However, the marginal fit gap among Group-2 (Vita In-Ceram YZ- Cerec ${ }^{\circledR}$ inLab) and group-3 (Aadva ${ }^{\mathrm{TM}}$ Zirconia-GM1000) specimens were $38.9 \pm 8.2 \mu \mathrm{m}$ and $20.8 \pm 8.3 \mu \mathrm{m}$ respectively. ANOVA showed a significant difference $(p<0.05)$ in marginal gap of restorations among the tested study groups. Group-II specimen showed significantly higher $(\mathrm{p}<0.05)$ marginal gap $(50.1 \pm 13.4 \mu \mathrm{m})$ than all other groups. Specimens in group-2 showed significantly lower $(p<0.05)$ marginal gap than Group-II and significantly higher $(\mathrm{p}<0.05)$ value compared to Groups three and four respectively (Table-I). Group-3 specimens showed lower $(\mathrm{p}<0.05)$ marginal gap than Groups one and two, however they showed higher $(p<0.05)$ marginal gap than Group-4 specimens. Group-3 and Group-4 specimens showed comparable $(p>0.05)$ outcomes for marginal gap of fixed partial denture frameworks.

The means and standard deviations for internal adaptation of frameworks in each group are presented in Table-I. The specimens in group-4 (Katana Zirconia-DWX-50N) showed the least gap $(40.2 \pm 8.8 \mu \mathrm{m})$ for internal adaptation, however Group-II (LAVA ${ }^{\mathrm{TM}}$ Zirconia- LAVA ${ }^{\mathrm{TM}}$ CNC 240) $(100.5 \pm 16.7 \mu \mathrm{m})$ showed the highest adaptation gaps among all groups. The internal adaptation values for group-2 and group-3 specimens were

Table-I: Comparison of means and standard deviations of Marginal Fit and internal adaptation $(\mu \mathrm{m})$ among study groups.

\begin{tabular}{lcccc}
\hline Study Groups & $\begin{array}{c}\text { Marginal Fit } \mu m \\
\text { Mean }(S D)\end{array}$ & $p_{\text {-value* }}^{*}$ & Internal Adaptation $(\mu m)$ & $p$-value \\
\hline Group-II & $50.1(13.4)^{\mathrm{a}}$ & & $100.5(16.7)^{\mathrm{a}}$ \\
Group-2 & $38.9(8.2)^{\mathrm{b}}$ & $<0.05$ & $102.5(13.4)^{\mathrm{a}}$ & \\
Group-3 & $20.8(8.3)^{\mathrm{c}}$ & $50.3(11.4)^{\mathrm{b}}$ & $<0.05$ \\
Group-4 & $16(4.0)^{\mathrm{c}}$ & $40.2(8.8)^{\mathrm{b}}$ & \\
\hline
\end{tabular}

Dissimilar superscript alphabets denote statistical different (Tukey-Kramer Test)-

* ANOVA. 
$102.5 \pm 13.4 \mu \mathrm{m}$ and $50.3 \pm 11.4 \mu \mathrm{m}$ respectively. Internal adaptation gap among group-3 (50.3 $\pm 11.4 \mu \mathrm{m})$ and $4(40.2 \pm 8.8 \mu \mathrm{m})$ specimens were statistically comparable $(\mathrm{p}>0.05)$. However, internal adaptation gap among group-3 $(50.3 \pm 11.4 \mu \mathrm{m})$ and $4(40.2 \pm 8.8 \mu \mathrm{m})$ specimens was significantly less $(\mathrm{p}<0.05)$ compared to Group-II $(100.5 \pm 16.7 \mu \mathrm{m})$ and group-2 (102.5 $\pm 13.4 \mu \mathrm{m})$ specimens respectively. Internal adaptation gap was comparable among Group-II (100.5 $\pm 16.7 \mu \mathrm{m})$ and group-2 (102.5 \pm 13.4 $\mu \mathrm{m})$ specimens $(\mathrm{p}>0.05)$ (Table-I).

\section{DISCUSSION}

The present study was based on the hypothesis, partially stabilized zirconia restorations when fabricated using different CAD-CAM milling systems will show a significant difference in their marginal fit and adaptation. It was observed that FPD fabricated with Katana Zirconia-DWX-50N (group-4) and Aadva ${ }^{\mathrm{TM}}$ Zirconia-GM1000 (group-3) showed better marginal fit and internal adaptation compared to LAVA ${ }^{\mathrm{TM}}$ Zirconia- LAVA ${ }^{\mathrm{TM}}$ CNC 240 (Group-II) and Vita In-Ceram YZ- Cerec ${ }^{\circledR}$ inLab (group-2) specimens. Therefore the hypothesis was accepted. A myriad of reasons are implicated for the findings including material properties, milling parameters (3 or 5 axis, soft or hard machining, burs size) and cement space and viscosity.

Multiple factors involved in the methodology of the study can influence the marginal gap and internal adaptation, including, cement type, cement thickness, cement mixing, seating pressure and preparation margin type. ${ }^{13} \mathrm{~A}$ standard resin cement (RelyX ${ }^{\mathrm{TM}}$ Unicem) was auto-mixed and FPDs were seated at a standard load of $50 \mathrm{~N}$ for 10 minutes for cementation. In addition, a deep chamfer margin was prepared for all specimens as it allows for thickness of ceramic and smooth flow and extrusion of luting cements. A single operator performed measurements of the fit and adaptation. Intra-examiner reliability was achieved after multiple measurements and a kappa score of 0.85 was achieved. Due to rapidly changing technology and materials for dental restorations and cements, there are no gold standards and guidelines for the acceptable clinical and biological fit and adaptation of indirect fixed partial dentures. The American Dental Association specification states that the luting cement film thickness for a crown restoration should be no more than $25 \mu \mathrm{m}$ using a type 1 luting agent or $40 \mu \mathrm{m}$ with a type II luting agent. ${ }^{14}$ Christensen (1966), in a classic study, reported that clinically detectable marginal discrepancy for sub-gingival margins is $34-119 \mu \mathrm{m}$, and $2-51 \mu \mathrm{m}$ for supra-gingival margins. ${ }^{15} \mathrm{He}$ also related, that marginal discrepancies of $39 \mu \mathrm{m}$ or more in visually accessible surfaces are unacceptable..$^{15}$ Some in vivo studies showed that clinically acceptable margins can range from 7-65 $\mu \mathrm{m} .{ }^{16}$ However, gaps of less than $80 \mu \mathrm{m}$ were proven to be very difficult to detect clinically, ${ }^{17}$ and several authors have considered that marginal discrepancies between $100-150 \mu \mathrm{m}$ to be clinically acceptable. ${ }^{18}$

In the present study, Aadva ${ }^{\mathrm{TM}}$ Zirconia $(\mathrm{Zr})$ frameworks milled by GM1000 and Katana Zirconia frameworks milled by DWX-50N were compared to LAVA $^{\mathrm{TM}}$ Zirconia frameworks milled by LAVA ${ }^{\mathrm{TM}}$ CNC 240 and Vita In-Ceram YZ frameworks milled by Cerec ${ }^{\circledR}$ inLab. There is limited data reporting the use of Katana zirconia and Aadva ${ }^{\mathrm{TM}}$ Zirconia in the literature. ${ }^{19}$ It was observed that the marginal gap widths for all tested materials and systems appeared to be considerably lower than what is reported in previous studies. ${ }^{20}$ In the present study, Vita In-Ceram YZ frameworks showed mean marginal gaps of $38.9 \pm 8.2 \mu \mathrm{m}$. In a similar study by Att et al (2009), mean marginal gap was $64 \mu \mathrm{m}$ for VITA In-Ceram YZ, which is considerably higher. ${ }^{20}$ In addition in the present study, group-4 (Katana Zirconia-DWX-50N) specimens showed lower marginal gap compared to specimens in Group-II (LAVA $^{\text {TM }}$ CNC 240). Katana Zirconia-DWX-50N milling machine is 5 -axis $(x, y, z, a, b)$ automatic tool changer (ATC) equipped with five milling tools capable of producing various shapes of zirconia restorations with undercuts. Its versatile 5 th axis allows materials to tilt as much as \pm 20 degrees. ${ }^{21}$ By contrast, Lava ${ }^{\mathrm{TM}} \mathrm{CNC} 240$ is a computer-controlled precision milling machine with three linear axes $(X$, $Y, Z$ ) and a rotational axis (A). Therefore a difference in milling axis may influence the milling accuracy. In addition, the type and number of burs employed in different milling systems may influence the precision milling ability of the systems resulting is compromise in marginal fit outcomes.

The present study revealed, that the internal adaptation of fixed partial denture specimens was significantly different based on the type of CADCAM system used. Specimens milled in group-3 (Aadva $^{\text {TM }}$ Zirconia GM1000) and group-4 (Katana Zirconia-DWX-50N) showed significantly lower internal adaptation gaps than Group-II (LAVA ${ }^{\mathrm{TM}}$ CNC 240) specimens. A possible explanation of these findings can be derived from the fact that LAVA Zr milling undergoes post milling sintering for milling (soft machining) efficiency of $\mathrm{Zr}$ substructure, 
resulting in a linear shrinkage of nearly $20 \% .{ }^{22}$ However there are compensatory mechanisms in the software and scanning process, sintering is a possible cause for increased discrepancies in the marginal fit and internal adaptation of zirconia frameworks, as shown in the present study. A systematic review by Abduo et al, concluded that $\mathrm{Zr}$ framework fit discrepancies are related to soft machining, curvatures and long framework spans. ${ }^{23}$ By contrast specimens in group- 3 and group -4 are milled from fully sintered $\mathrm{Zr}$ blocks. Moreover, the specimens in group- 3 were fabricated with GM 1000 , a 5 -axis laser milling system. It is suggested previously, that a 3-axis milling system (LAVA CNC 240) shows insufficient accuracy of longitudinal milling in comparison to 5-axis laser milling, therefore resulting in higher internal adaptation gaps for $\mathrm{Zr}$ frameworks. ${ }^{19}$ These findings of better fit and adaptation of $\mathrm{Zr}$ frameworks made from 5 -axis laser milling compared to a 3 to 4 axis milling are in line with a previous study. ${ }^{19}$ Therefore a 5 -axis laser milling system for post sintered $\mathrm{Zr}$ is recommended for frameworks on abutments of complex anatomy and tall height with teeth and dental implants. Furthermore, other factors such as bur size, dry and wet milling, cement space and operator experience are some factors which can be implicated for the observations in the present study. ${ }^{23-24}$

Limitations of the study: Outcomes of the present study should be interpreted in light of the limitations. The $\mathrm{Zr}$ materials used in the study groups were manufactured by different companies, therefore may differ in composition. In addition, FPD frameworks were seated on artificial teeth abutments with a static force of $50 \mathrm{~N}$, however intraoral forces are dynamic and can be upto $400 \mathrm{~N}$ in some cases. The cemented specimens were sectioned using a destructive method, which may have possibly influenced the fit and adaptation measurement outcomes. Hard machining with a 5-axis milling although showed better fit and adaptation outcomes for $\mathrm{Zr}$ frameworks in the present study, however there is a continuous need for further improvements in the technology of hard machining with regards to cutting efficiency, time consumption and surface properties of milled materials. In addition, the introduction of additive technology (3D Printing) for the manufacture of metal and $\mathrm{Zr}$ frameworks of dental restorations provide improvement potential in restorative fit and adaptation. Therefore, further investigations on the properties of multiple axis, laser milled and printed, hard machined Zr framework including surface topography, flexural strength, fracture toughness and color stability are recommended.

\section{CONCLUSION}

The Zr CAD-CAM systems assessed exhibited marginal fit and internal adaptations measurements of 16.0 to $50.1 \mu \mathrm{m}$ and 40.2 to $102.5 \mu \mathrm{m}$ respectively. For both fit and adaptation, specimens in Group-3 (Aadva $^{\mathrm{TM}}$ Zirconia (Zr)- GM1000) and Group-4 (Katana Zirconia- DWX-50N) showed better outcomes then specimens in Group-II (LAVA ${ }^{\mathrm{TM}}$ Zirconia/ LAVA ${ }^{\mathrm{TM}} \mathrm{CNC} 240$ ) and group-2 (Vita InCeram YZ/ Cerec $®$ inLab). Marginal fit and internal adaptation values of all materials and systems tested were within clinically acceptable standards.

Acknowledgement: The authors are grateful to the Deanship of Scientific Research, King Saud University for funding through Vice Deanship of Scientific Research Chairs, Research Chair for Biological Research in Dental Health.

Conflict of Interest: The authors declare no conflict of interests

\section{REFERENCES}

1. Heffernan MJ, Aquilino SA, Diaz-Arnold AM, Haselton DR, Stanford CM, Vargas MA. Relative translucency of six all-ceramic systems. Part I: Core materials. J Prosth Dent. 2002;88(1):4-9. doi: 10.1067/mpr.2002.126794

2. Miyazaki T HY, Kunii J, Kuriyama S, Tamaki Y. A review of dental CAD:CAM current status and future perspectives from 20 years of experience. Dent Mater J. 2009;28(1):44-56. doi: $10.4012 / \mathrm{dmj} .28 .44$

3. Al Deeb L, Al Ahdal K, Alotaibi G, Alshehri A, Alotaibi B, Alabdulwahab F, et al. Marginal Integrity, Internal Adaptation and Compressive Strength of 3D Printed, Computer Aided Design and Computer Aided Manufacture and Conventional Interim Fixed Partial Dentures. J Biomater Tissue Eng. 2020;9(12):1745-1750.

4. Ender A, Mehl A. CEREC Basic Information. A Clinical Guide 2007. http:/ / www.sirona.com/ecomaXL/get_blob. php?name=CEREC_Basic_Information_3.8.pdf

5. Sindel J, Petschelt A, Grellner F, Dierken C, Greil P. Evaluation of subsurface damage in CAD/CAM machined dental ceramics. J Mater Sci Mater Med. 1998;9(5):291-295. doi: 10.1023/a:1008812929476

6. Sorensen SE, Larsen IB, Jorgensen KD. Gingival and alveolar bone reaction to marginal fit of subgingival crown margins. Scand J Dent Res. 1986;94(2):109-114. doi: 10.1111/j.1600-0722.1986.tb01373.x

7. Lillywhite GR, Vohra F. Influence of polyurethane resin dies on the fit and adaptation of full veneer crowns. Indian J Dent Res. 2015;26(1):72.

8. Shim JS, Lee JS, Lee JY, Choi YJ, Shin SW, Ryu JJ. Effect of software version and parameter settings on the marginal and internal adaptation of crowns fabricated with the CAD/CAM system. J Applied Oral Sci. 2015;23(5):515-522. doi: 10.1590/1678-775720150081 
9. Hamza TA, Sherif RM. In vitro evaluation of marginal discrepancy of monolithic zirconia restorations fabricated with different CAD-CAM systems. J Prosthet Dent. 2017;117(6):762-766. doi: 10.1016/j.prosdent.2016.09.011

10. Hamza TA, Ezzat HA, El-Hossary MM, Katamish HA, Shokry TE, Rosenstiel SF. Accuracy of ceramic restorations made with two CAD/CAM systems. J Prosthet Dentist. 2013;109(2):83-87. doi: 10.1016/S0022-3913(13)60020-7

11. Atlas A, Isleem W, Bergler M, Fraiman HP, Walter R, Lawson ND. Factors Affecting the Marginal Fit of CADCAM Restorations and Concepts to Improve Outcomes. Current Oral Health Reports. 2019;6(4):277-283. doi: 10.1007/s40496-019-00245-2

12. Kirsch C, Ender A, Attin T, Mehl A. Trueness of four different milling procedures used in dental CAD/CAM systems. Clin Oral Investig. 2017;21(2):551-558. doi: 10.1007/s00784-016-1916-y

13. Abduo J, Lyons K, Bennamoun M. Trends in computeraided manufacturing in prosthodontics: a review of the available streams. Int J Dent. 2014;2014:783948. doi: $10.1155 / 2014 / 783948$

14. Association AD. ANSI/ADA Specification No. 8 for zinc phosphate cement. In Guide to Dental Materials and Devices (ed 5). 1970-1971:87-88. doi: 10.6028/nbs.sp.571

15. Christensen GJ. Marginal fit of gold inlay castings. J Prosth Dent. 1966;16(2):297-305. doi: 10/1016/00223913(66) $90082-5$

16. Lofstrom LH, Barakat MM. Scanning electron microscopic evaluation of clinically cemented cast gold restorations. J Prosth Dent. 1989;61(6):664-669. doi: 10.1016/s00223913(89)80038-1

17. McLean JW, von Fraunhofer JA. The estimation of cement film thickness by an in vivo technique. Br Dent J. 1971;131(3):107-111. doi: 10.1038/sj.bdj.4802708

18. Boening KW, Walter MH, Reppel PD. Non-cast titanium restorations in fixed prosthodontics. J Oral Rehabil. 1992;19(3):281-287. doi: 10.1111/j.1365-2842.1992.tb01103.x

19. Ohkuma K, Kameda T, Terada K. Five-axis laser milling system that realizes more accurate zirconia CAD/CAM crowns by direct milling from fully sintered blocks. Dent Mater J. 2019;38(1):52-60. doi: 10.4012/dmj.2017-443

20. Att W, Komine F, Gerds T, Strub JR. Marginal adaptation of three different zirconium dioxide three-unit fixed dental prostheses. J Prosth Dent. 2009;101(4):239-247. doi: 10.1016/S0022-3913(09)60047-0

21. https://www.aegisdentalnetwork.com/id/products/ noritake-dental-supply-co-limited/katana-millingmachine-dwx-50n.
22. Samer MS, Faraz Q, Al-Dubai SA, Vohra F, Abdullah H, Taiyeb-Ali TB, Saub R. Clinical outcomes and predictors of satisfaction in patients with improved lithium disilicate all-ceramic crowns. Med Princ Pract. 2017;26(5):470-479. doi: $10.1159 / 000481864$

13. Abduo J, Lyons K, Bennamoun M. Trends in computeraided manufacturing in prosthodontics: a review of the available streams. Int J Dent. 2014;2014:783948. doi: $10.1155 / 2014 / 783948$

23. Ortorp A, Jonsson D, Mouhsen A, von Steyern PV. The fit of cobalt-chromium three-unit fixed dental prostheses fabricated with four different techniques: A comparative in vitro study. Dent Mater. 2011;27(4):356-363. doi: 10.1016/j. dental.2010.11.015

24. Bornemann G. Innovative method for the analysis of the internal 3D fitting accuracy of Cerec-3 crowns. Int J Comput Dent. 2002;5:177-182.

\section{Authors' Contribution:}

KA: Experimentation, Data collection, study design, manuscript writing, final manuscript approval.

AM: Data collection, study design, manuscript drafting, data analysis, funding and equipment, manuscript approval.

RA: Specimen design and preparation, Data collection, manuscript approval and data interpretation.

FV \& TA: Data collection, sample preparation, writing, revision, editing, final manuscript approval, table and figure designing.

As per the ICMJE authorship requirement, all authors are responsible and accountable for the accuracy or integrity of the work.

Authors:

1. Khulud AAl-Aali

Department of Clinical Dental Sciences,

2. Rana S Alhamdan

Department of Restorative Dental Sciences,

3. Ahmed M Maawadh

Department of Restorative Dental Sciences,

4. Fahim Vohra

Department of Prosthetic Dental Science,

5. Tariq Abduljabbar

Department of Prosthetic Dental Science,

1: College of Dentistry,

Princess Nourah Bint Abdulrahman University,

Riyadh, Saudi Arabia.

2, 3: College of Dentistry,

King Saud University; Riyadh 11545,

Saudi Arabia.

4, 5: College of Dentistry,

King Saud University,

Riyadh 11545, Saudi Arabia.

Research Chair for Biological Research in Dental Health,

King Saud University,

Riyadh, Saudi Arabia. 